\title{
A Micro-Canonical Description of Hadron Production in Proton-Proton Collisions
}

\author{
F. M. Liu ${ }^{1,2,3 *}$ K. Werner ${ }^{3}$, J. Aichelin ${ }^{3}$, M. Bleicher ${ }^{2}$, H. Stöcker ${ }^{2}$
}

7th July 2018

\author{
${ }^{1}$ Institute of Particle Physics, Central China Normal University, Wuhan, China \\ ${ }^{2}$ Institut fuer Theoretische Physik, J. W. Goethe-Universitaet, Frankfurt am Main, Germany \\ ${ }^{3}$ Laboratoire SUBATECH, Universite de Nantes - IN2P3/CNRS - Ecole des Mines de Nantes, \\ Nantes, France
}

\begin{abstract}
A micro-canonical treatment is used to study particle production in pp collisions. First this micro-canonical treatment is compared to some canonical ones. Then proton, antiproton and pion $4 \pi$ multiplicities from proton-proton collisions at various center of mass energies are used to fix the micro-canonical parameters $E$ and $V$. The dependences of the micro-canonical parameters on the collision energy are parameterised for the further study of pp reactions with this micro-canonical treatment.
\end{abstract}

In recent years, the use of thermal statistical models to describe particle ratios for heavy ion collisions has received great attention 1, 2, 3, 4, 5, 6, 7, 8, Thermal models are even used to describe protonproton and $\mathrm{e}^{+} \mathrm{e}^{-}$collisions. The corresponding volumes for these reactions are very small $(V \sim$ $25 \mathrm{fm}^{3}$ ). For small volumes, energy and flavour conservation become important. Therefore a microcanonical treatment should be employed. With the increase of the volume, the micro-canonical results should converge towards the canonical one.

The following micro-canonical treatment [9] is employed. Following the general philosophy of statistical approaches to hadron production, we suppose that the result of a high energy collision can be considered as a distribution of "clusters", "droplets", or "fireballs", which move relative to each other. Here, we are only interested in $4 \pi$ particle yields and average transverse momenta, and the distribution of clusters may be identified with one single "equivalent cluster", being characterised by its volume $V$ (the sum of individual proper volumes), its energy $E$ (the sum of all the cluster masses), and the net flavour content $Q=\left(N_{u}-N_{\bar{u}}, N_{d}-N_{\bar{d}}, N_{s}-N_{\bar{s}}\right)$. The basic assumption is that a cluster, characterised by $V, E$, and $Q$, decays "statistically" according to phase space. More precisely, the probability of a cluster to hadronize into a configuration $K=\left\{h_{1}, \ldots, h_{n}\right\}$ of hadrons $h_{i}$ is given by the micro-canonical partition function $\Omega(K)$ of an ideal, relativistic gas of the $n$ hadrons $h_{i}$,

$$
\Omega(K)=\frac{V^{n}}{(2 \pi \hbar)^{3 n}} \prod_{i=1}^{n} g_{i} \prod_{\alpha \in \mathcal{S}} \frac{1}{n_{\alpha} !} \int \prod_{i=1}^{n} d^{3} p_{i} \delta\left(E-\Sigma \varepsilon_{i}\right) \delta\left(\Sigma \vec{p}_{i}\right) \delta_{Q, \Sigma q_{i}},
$$

with $\varepsilon_{i}=\sqrt{m_{i}^{2}+p_{i}^{2}}$ being the energy, and $p_{i}$ being the 3 -momentum of particle $i$. The term $\delta_{Q, \Sigma q_{i}}$ ensures flavour conservation; $q_{i}$ is the flavour vector of hadron $i$. The symbol $\mathcal{S}$ represents the set of hadron species considered: we take $\mathcal{S}$ to contain the pseudoscalar and vector mesons $\left(\pi, K, \eta, \eta^{\prime}, \rho, K^{*}, \omega, \phi\right)$ and the lowest spin- $\frac{1}{2}$ and spin- $\frac{3}{2}$ baryons $\left(N, \Lambda, \Sigma, \Delta, \Sigma^{*}, \Xi^{*}, \Omega\right)$ and the

*Fellow of the Alexander von Humboldt Foundation 
corresponding antibaryons. $n_{\alpha}$ is the number of hadron species $\alpha$, and $g_{i}$ is the degeneracy of particle $i$.

For the present investigation, we limit the number of hadrons in $\mathcal{S}$ to 54 . Strange particles are produced according to phase space, i.e. without applying any artificial suppression factor. The results are compared to that of the canonical calculations using the approach of Becattini et al. [10, 12, 11], but without strangeness suppression and the number of hadrons which can be produced is limited to the same 54 species allowed in our micro-canonical treatment. In principle it is possible to include more hadrons in the micro-canonical ensemble. This makes a detailed comparison more difficult, because the less known decay channels of the additional hadrons have to agree.

For micro-canonical treatment, there exists a critical volume $V_{c}$, above which the physics results, i.e. particle yield per volume and average transverse momentum, become independent of volume and coincide to the canonical ones. In the following we check the volume effect in particle yields, compare the micro-canonical results to canonical one and try to find the critical volume $V_{c}$. To do this calculation, we keep the same baryon density as input, i.e. the baryon number is two (a proton-proton collision) for the volume $25 \mathrm{fm}^{3}$ which is usually used by canonical calculations (dashed lines), baryon number one for volume $12.5 \mathrm{fm}^{3}$ (solid lines) and baryon number four for volume $50 \mathrm{fm}^{3}$ (dotted lines), c.f. Figs 11 2] 3. The results of a canonical calculation with the parameters fitted to describe the particle yields observed in pp collisions at $27.4 \mathrm{GeV}$ [10, 12, 11] are marked as dot points. The parameters used in this canonical calculations are $V=25.5 \mathrm{fm}^{3}$ and $T=162 \mathrm{MeV}$. In contradistinction to the calculation with this parameter which is presented in ref. 11 here the strange particles are not suppressed by a $\gamma_{s}$ factor and only the 54 hadron species mentioned above are produced. Therefore this canonical calculation can directly be compared with our micro-canonical approach. The average energy of the clusters obtained in this calculation is $8.74 \mathrm{GeV}$, resulting in an average energy density of $\varepsilon=0.342 \mathrm{GeV} / \mathrm{fm}^{3}$. More detailed explanation of Figs 11, 23] can be found in 13 .

Figs 1 2 3 tell us, the lighter is the partcile, the smaller is the critical volume $V_{c}$. 90\% newlyproduced hadrons from the collisions are pions while the critical volume $V_{c}$ is not bigger than $12.5 \mathrm{fm}^{3}$. However, for the heavy particles, i.e. $\Delta^{++}$and $\Omega$, the critical volume $V_{c}$ is bigger than $50 \mathrm{fm}^{3}$. The canonical results agree well with the micro-canonical one at the same volume, $25 \mathrm{fm}^{3}$. Therefore we raise the question if it is safe to study the heavy particles from small reaction systems with canonical models.

Canonical calculations get volume $25 \mathrm{fm}^{3}$ from fitting particle yields. Here we also fit the $4 \pi$ particle yields from pp collisions to fix the micro-canonical parameters $E$ and $V$. The micro-canonical model does not have any strangeness suppression factor. It fails to describe non-strange and strange hadrons at the same time. So we only consider non-strange hadron production here. To fit the data, we minimize

$$
\chi^{2}=\frac{1}{\alpha} \sum_{j=1}^{\alpha} \frac{\left[n_{\mathrm{exp}, j}(\sqrt{s})-n_{\mathrm{th}, j}(E, V)\right]^{2}}{\sigma_{j}^{2}}
$$

to determine the parameter $E$ and $V$ for at each collision energy $\sqrt{s}$, where $n_{\exp , j}, n_{\mathrm{th}, j}$ and $\sigma_{j}$ are respectively the experimental multiplicity, multiplicity from the micro-canonical treatment and experimental variance of particle $j$. Both the experimental and theoretical multiplicities here are after decays. We take the parameterization[14] of energy dependence of $\pi^{+}, \pi^{-}$, proton and antiproton instead of the data to fix the micro-canonical parameters $E$ and $V$ for a convenient reason. The minimum $\chi^{2}$ method produces the micro-canonical parameters $E$ and $V$ dependence on collision energy $\sqrt{s}$ as shown in Fig. 4. $E$ and $V$ increase monotonously with $\sqrt{s}$. We parameterize the micro-canonical parameters $E(\mathrm{GeV})$ and $V\left(\mathrm{fm}^{3}\right)$ dependence on the collision energy $\sqrt{s}(\mathrm{GeV})$ for some further study of pp cillisions with this canonical treatment:

$$
\begin{aligned}
& E=-3.8+3.76 \ln \sqrt{s}+6.4 / \sqrt{s} \\
& V=-30.04+14.93 \ln \sqrt{s}-0.013 \sqrt{s}
\end{aligned}
$$

Note that $V_{\min }=1 \mathrm{fm}^{3}$ has been used for Eq. 2] at very low energies. The Eq. 11itself is the same as charged hadron excitation function in pp collisions [14]. This agrees well with the results in heavy ion 

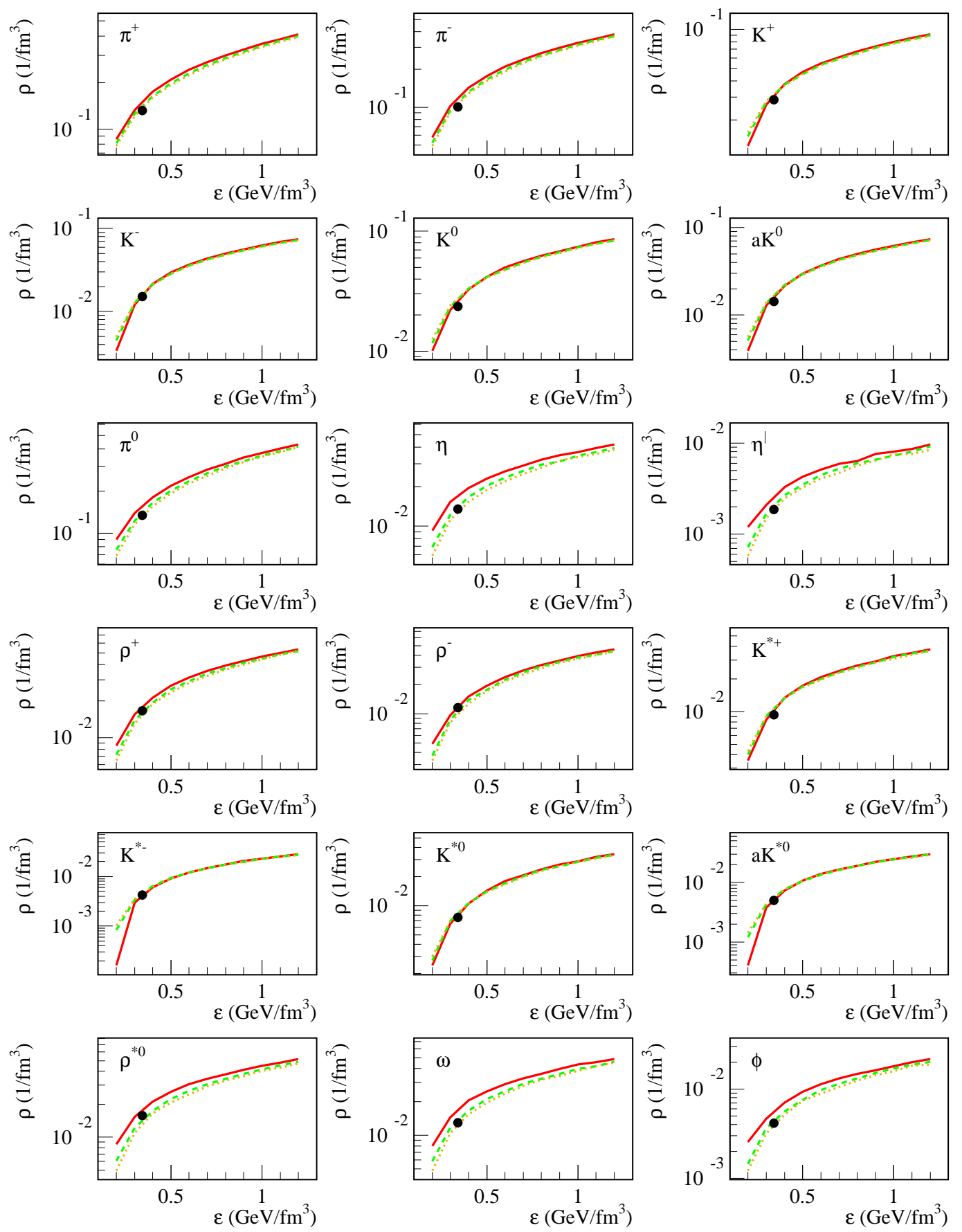

Figure 1: Density of particles as a function of the energy density of a cluster for three different volumes: $12.5 \mathrm{fm}^{3}$ (dashed line) and $50 \mathrm{fm}^{3}$ and a total charge of 2 using a micro-canonical phase space calculation. The dots present the result of a canonical calculation [10, 12, 11, provided by Becattini. In both cases the number of hadrons is limited to 54 and strange particles are not suppressed. Taken from 13 . 

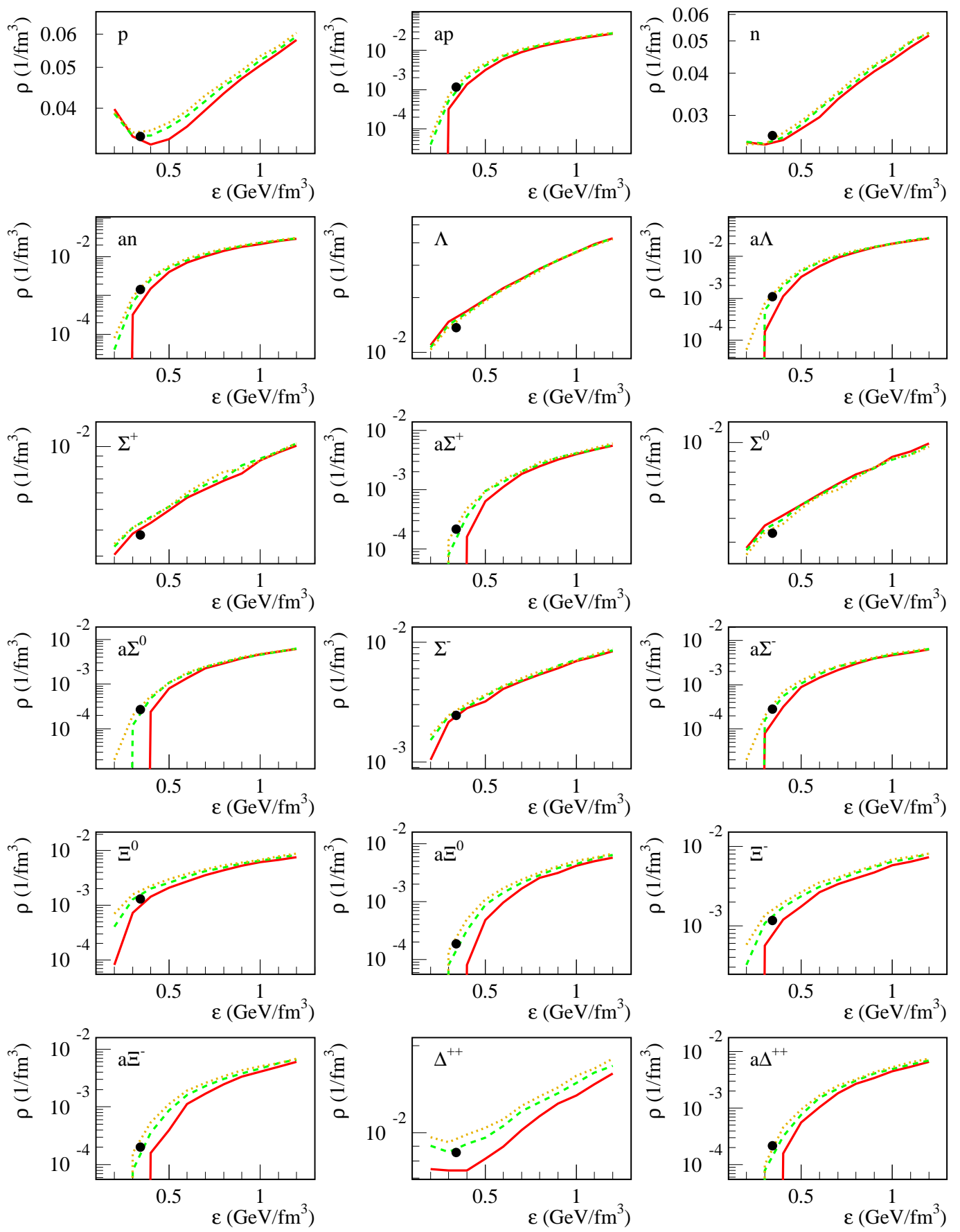

Figure 2: Same as Fig. 1 but for additional hadrons 

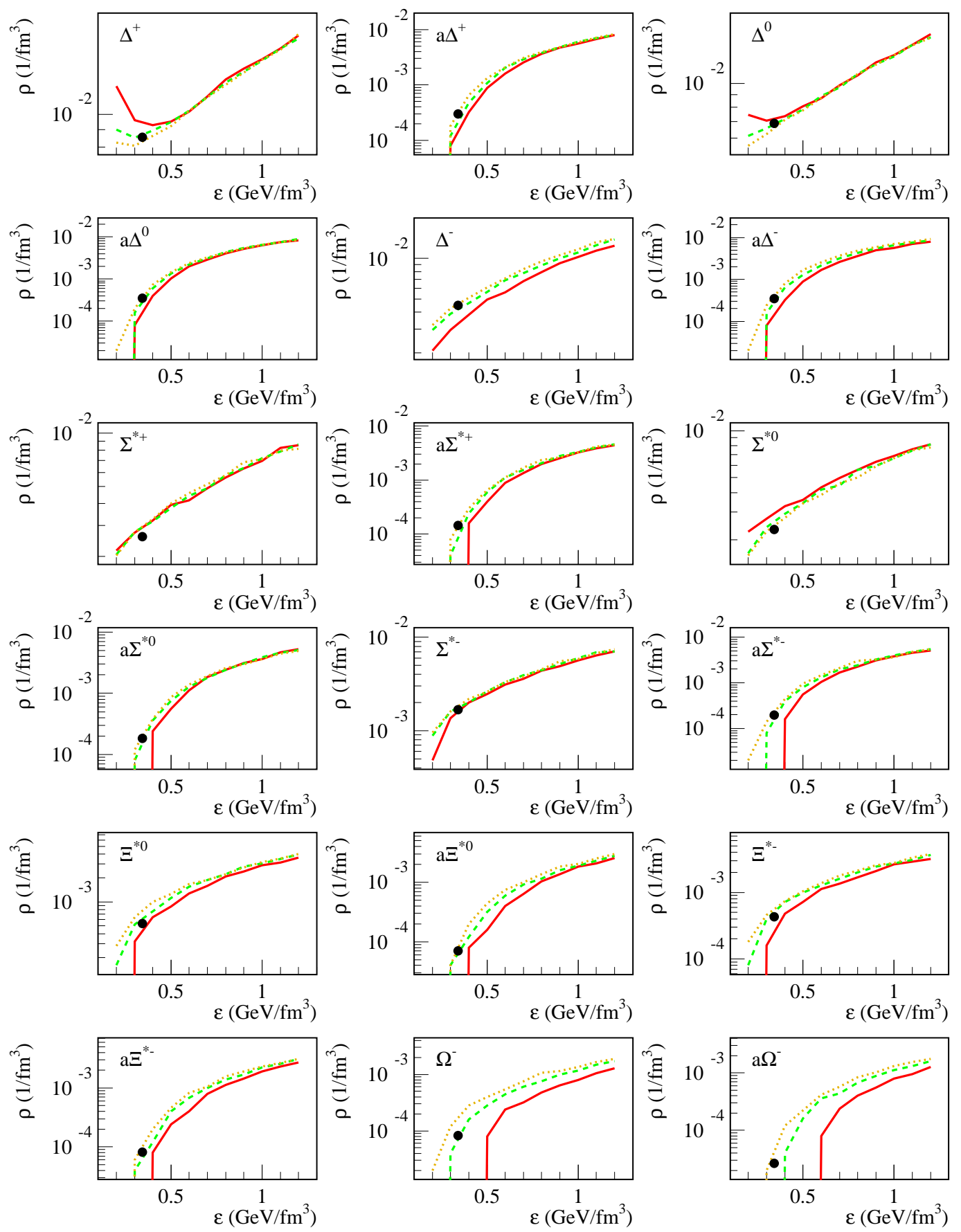

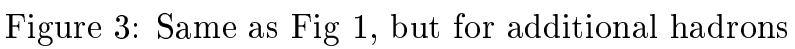



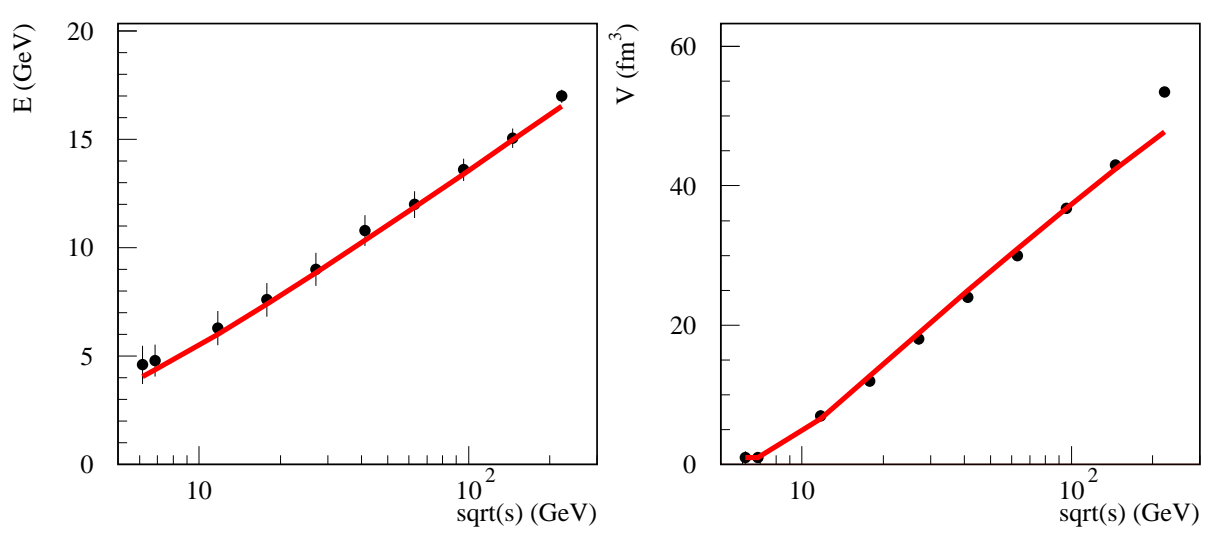

Figure 4: Micro-canonical parameters $E$ (left) and $V$ (right) dependence on the collision energy $\sqrt{s}$. The points are from fitting. The solid lines are the parameterized function described in Eq. 1 and 2

collsions 15. where the freeze-out energy per particle $\langle E\rangle /\langle N\rangle=1 \mathrm{GeV}$, taking into account that the increase of the multiplicity from decay has roughly the same fraction of neutral particles in the total hadron multiplicity.

Acknowledgment

We would like to thank F. Becattini for many discussions and for providing us with the calculation with a restricted set of hadrons and without strangeness suppression.

\section{References}

[1] E. Fermi, Prog. Theor. Phys. 5, 570 (1950); Phys.Rev. 81 (1951) 683.

[2] L. D. Landau, Lzv. Akd. Nauk SSSR 17 (1953) 51; Collected papers of L. D. Landau, ed. D. Ter Haar, Gordon and Breach, New York, 1965

[3] R. Hagedorn, Nucl. Phys. B 24 (1970) 93.

[4] P. Siemens, J. Kapusta, Phys. Rev. Lett. 43 (1979) 1486.

[5] A. Z. Mekjian, Nucl. Phys. A384 (1982) 492.

[6] L. Csernai, J. Kapusta Phys. Rep. 131 (1986) 223.

[7] H. Stoecker, W. Greiner, Phys. Rep. 137 (1986) 279.

[8] J. Rafelski and J. Letessier, J. Phys. G: Nucl. Part. Phys. 28 (2002) 1819-1832.

[9] K. Werner and J. Aichelin, Phys. Rev. C52 (1995) 1584-1603.

[10] F. Becattini, G. Passaleva, Eur.Phys.J. C23 (2002) 551

[11] F. Becattini, U. Heinz Z.Phys. C76 (1997) 269-286

[12] F. Becattini , A. Giovannini, S. Lupia Z.Phys. C72 (1996) 491

[13] F.M. Liu, K. Werner, J. Aichelin, hep-ph/0304174 Comparison of micro-canonical and Canonical Hadronization.

[14] Antinucci et al, Letters al Nuovo Cimento, V6, N4, 27 Gennaio1973.

[15] J. Cleymans and K. Redlich, Phys.Rev.Lett. 81:5284-5286,1998. 